\title{
Publisher Correction: What we know and what we don't know about the proton spin after 30 years
}

\author{
Xiangdong Ji(D), Feng Yuan (D) and Yong Zhao
}

Correction to: Nature Reviews (2020) https://www.nature.com/articles/s42254-020-00248-4, published online 23 November 2020.

In the originally published article eq 6 contained incorrect superscripts and subscripts which have now been corrected. In the text after eq 3 , D should have been slashed D. In eq 9 a new line was added after the comma to better show the two equations. In eq 18 the definition of the limit was not subscripted correction and it has now been corrected. The editors apologise for these issues.

https://doi.org/10.1038/s42254-020-00274-2 I Published online 21 December 2020

(c) Springer Nature Limited 2020 